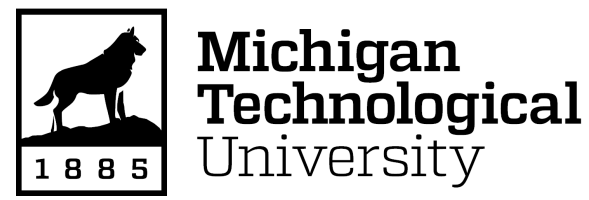

Michigan Technological University Digital Commons @ Michigan Tech

Dissertations, Master's Theses and Master's Reports

2020

\title{
3D PRINTING OF IRON OXIDE INCORPORATED POLYDIMETHYLSILOXANE SOFT MAGNETIC ACTUATOR
}

Rasoul Bayaniahangar

Michigan Technological University, rbayania@mtu.edu

Copyright 2020 Rasoul Bayaniahangar

\section{Recommended Citation}

Bayaniahangar, Rasoul, "3D PRINTING OF IRON OXIDE INCORPORATED POLYDIMETHYLSILOXANE SOFT MAGNETIC ACTUATOR", Open Access Master's Thesis, Michigan Technological University, 2020.

https://doi.org/10.37099/mtu.dc.etdr/1016

Follow this and additional works at: https://digitalcommons.mtu.edu/etdr

Part of the Biomaterials Commons, Biomedical Devices and Instrumentation Commons, Manufacturing Commons, and the Nanoscience and Nanotechnology Commons 
3D PRINTING OF IRON OXIDE INCORPORATED POLYDIMETHYLSILOXANE SOFT MAGNETIC ACTUATOR

\author{
By \\ Rasoul Bayaniahangar
}

\begin{abstract}
A THESIS
Submitted in partial fulfillment of the requirements for the degree of MASTER OF SCIENCE

In Mechanical Engineering
\end{abstract}

MICHIGAN TECHNOLOGICAL UNIVERSITY

2020

(C) 2020 Rasoul Bayaniahangar 
This thesis has been approved in partial fulfillment of the requirements for the Degree of MASTER OF SCIENCE in Mechanical Engineering.

Department of Mechanical Engineering-Engineering Mechanics

Thesis Co-Advisor: $\quad$ Joshua M. Pearce

Thesis Co-Advisor: $\quad$ Craig Friedrich

Committee Member: $\quad$ Patricia A. Heiden

Department Chair: William Predebon 


\section{Table of Contents}

Acknowledgements ............................................................................................... iv

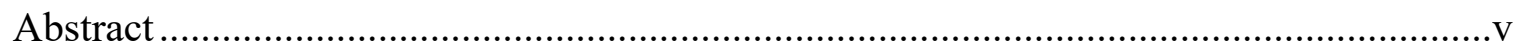

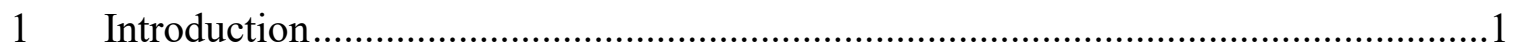

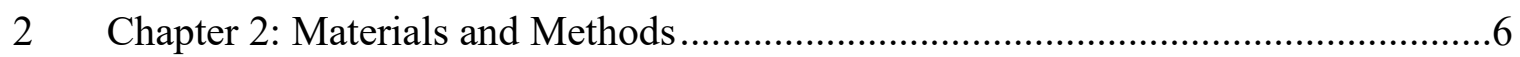

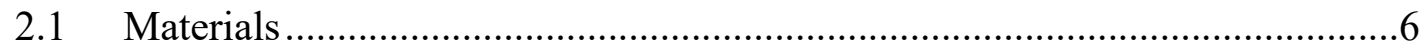

2.2 Preparation of PDMS- Iron Oxide Nanoparticle Composite.............................6

2.3 Preparation of Pluronic Hydrogel.............................................................

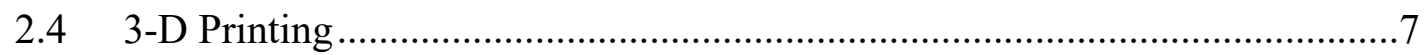

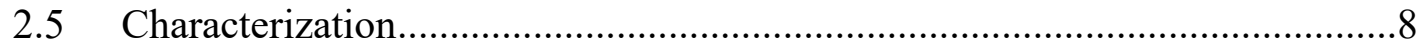

2.5.1 Printing Material Rheometry ………………..............................

2.5.2 Microscopic Imaging ................................................................

2.5.3 Atomic force microscopy …….........................................................

2.5.4 Linear and Bending magnetic actuation test......................................

2.5.5 Coil Locomotion Tests..................................................................10

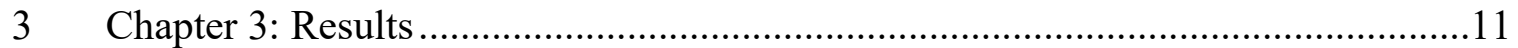

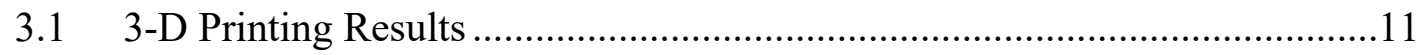

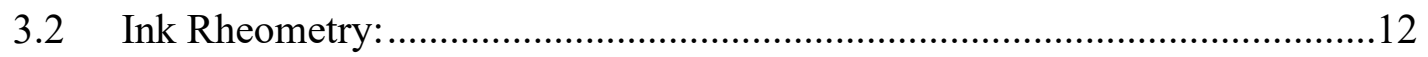

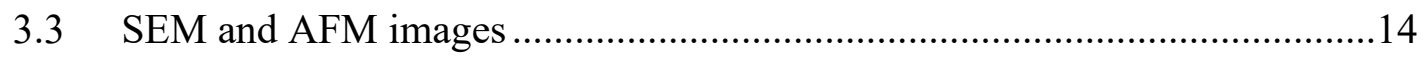

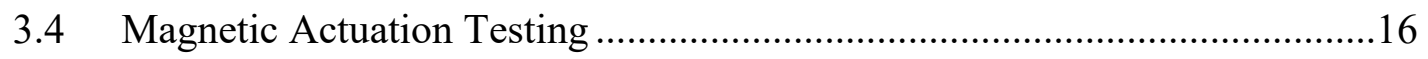

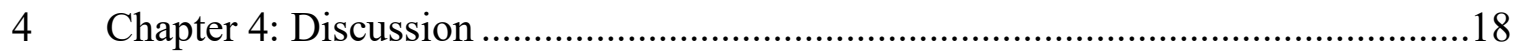

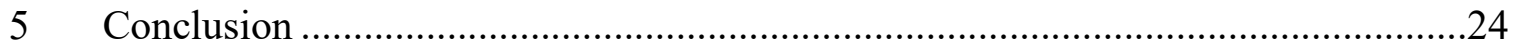

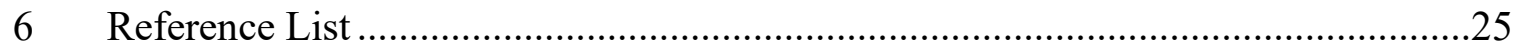




\section{Acknowledgements}

I am sincerely grateful to Dr. Craig Friedrich for being my coadvisor. I would like to express my gratitude to Dr. Joshua Pearce who gave me the chance of working and learning under him. Working with him was a memorable part of my Michigan Tech journey that was filled with surprise and joy, the unique experience of how much doing a graduate school could be delightful. I would like to sincerely thank Dr. Patricia Heiden who always believed in me and supported me, enlightening the right path to take and giving me the courage to take that. Not only she is my mentor but also a role model for me, not just in the academic and professional life, but more importantly in always trying to be a better person.

I would like to thank my parents, Shahla and Alireza for all the sacrifices they have done throughout my life to help me reach the place I am. I would like to thank my sister Shohreh that her being in this very world is always a piece of mind to me. I would like to thank my brother Shahab, who also is $\mathrm{PhD}$ student in mechanical engineering at MTU. He was the first teacher I had in my life, clearly remembering him teaching me how to play chess when I was 4 . He was my teacher throughout my life in mid-school, high school, college days and even till few days ago in this very project, giving brilliant ideas in how to improve this work. And, last but not least, I would like to thank Sahar, the old-time friend of mine, who I grow up with her and spent best days of my life with. 


\section{Abstract}

Soft actuators have grown to be a topic of great scientific interest recently. As the fabrication of soft actuators with conventional microfabrication methods are tedious, expensive, and time consuming, employment of 3-D printing fabrication methods appears promising as they can simplify fabrication and reduce the production cost. Complex structures can be fabricated with 3-D printing such as helical coils can achieve actuation performances that otherwise would not be possible with simpler geometries. In this thesis development of soft magnetic helical coil actuators of iron-oxide embedded polydimethylsiloxane (PDMS) was achieved with embedded 3-D printing techniques. Composites with three different weight ratios of $10 \%, 20 \%$, and $30 \%$ iron nanoparticles to PDMS were formulated. Using iron nanoparticles with 15-20nm size helps preserve viscosity of the printing material low enough that it was possible to print it with small gauge 29 needle (180 micrometers inner diameter). The hydrogel support of Pluroic f-127 bath and the ability to maintain the ratio of the printed fiber's diameter to coil diameter close to 0.25 approximately resulted in the successful fabrication and release of fabricated helical coil structures. This enabled 3-D printed structures characterized as magnetic actuators to achieve linear and bending actuation of more than $300 \%$ and $80^{\circ}$ respectively in the case of composites with $30 \%$ iron oxide nanoparticles. Moreover, it was shown that the $3 \mathrm{D}$ printed helical coils with $10 \%$ iron oxide nanoparticles can be utilized as untethered soft robot that is capable of locomotion on 45 and 90 degrees inclines under an applied magnetic field. 


\section{Introduction}

Actuators are devices that can deform in response to an applied stimuli. Various types of stimuli have been utilized for actuators including: current, pressure, temperature, voltage, light, and magnetic fields.[1,2] Depending on the type of applied stimuli, actuators have various applications in the automotive, aerospace, biomedical, and robotic industries. Soft actuators, are a special class of actuators made of soft materials which have been of great interest recently for possible applications in microfluidic systems, medical devices for minimally invasive surgery, rehabilitation, locomotion, manipulation of fragile agriculture products, human-machine interfaces, and smart textiles[3-5]. Fabrication of these soft actuators through conventional fabrication techniques requires tedious and timeconsuming iteration of molding, lithography, and post-processing, which increases the costs and limits the complexity of the fabricated actuator's structures.[6] Recently, the radical cost drop in 3-D printing technology because of the open source release of material extrusion 3-D printers[7, 8] and subsequent evolution[9-11] has made soft actuator fabrication far more accessible.[12-15] 3-D printing techniques enables simpler fabrication of complex actuators in one step without the need for post-processing and reduces fabrication time and waste material which in turn eliminates the high cost of fabrication and labor.[16]Moreover, fabrication of structures in one step instead of assembling them could potentially enhance their performance.[17]

Magnetic fields are among the types of stimulus that can be remotely applied to materials with ferromagnetic parts or particles to create soft actuators. Furthermore, magnetic fields provide a relatively fast response[18] and the deformation is reversible. 
Soft actuators mostly consist of polymers, hydrogels, and elastomers as the matrix materials with embedded magnetic particles as the filler materials Iron oxide $\left(\mathrm{Fe}_{3} \mathrm{O}_{4}\right)[19$, 20], neodymium-iron-boron (NdFeB)[21], and paraseodymium-iron-boron ( $\mathrm{PrFeB})[22]$ have been used as the filler materials. While the added particles reduce the flexibility of the composite, response of these particles to the applied magnetic field results in small scale forces (and torques) on each particle that eventually deform the soft composite. Pirmoradi et al. fabricated a magnetic membrane of iron oxide nanoparticles in polydimethylsiloxane (PDMS) matrix using microfabrication techniques and characterized it as an actuator.[23] Evans et al. synthesized core-shell particles of iron oxide magnetic particles with PDMS complex to fabricate a magnetic membrane.[24] Electrically conductive composites were fabricated using silver coated magnetic particles dispersed in PDMS matrix where curing under an applied magnetic field caused alignment of the particles along the constant magnetic field direction, and it was shown that the composite's resistivity will change under applied pressure or magnetic field.[25] Magnetic film had also been fabricated using PDMS and magnetic particles and their application had been studied as the valve in microfluidic devices.[26]

Bioinspired soft robots in the form of inchworm, turtle, and millipede were fabricated using magnetic microparticles in silicon rubber and their locomotion mechanisms were studied with actuation under applied magnetic field.[22, 27, 28] Most of the structures fabricated through these techniques were either simple structures such as films, while more complicated structures were fabricated through multiple microfabrication techniques and assemblies. 
3-D printing enables the fabrication of more complex structures in a simpler one step and some research has been done in this area for magnetic actuators. An early trial on inkjet printing of a material to develop magnetic structures was done with a custom-made 3-D printing stage, where the iron oxide magnetic particles were dispersed in a solvent and by evaporating the solvent, simple structures as rings and squares were formed.[29] Zhang et al. formulated filaments of polylactic acid (PLA) with dispersed iron oxide particles composite and 3-D printed various structures with them. The shape memory effect of these structures was used for actuation. By applying an alternating magnetic field at the high frequency of $25-50 \mathrm{kHz}$, magnetic particles vibration generated heat and introduced shape change. As the magnetic field was removed, the generated heat was lost and structures returned to their original shape.[30] Zhu et al. developed a novel artificial microfish of poly(ethylene glycol) diacrylate (PEGDA) hydrogel with added iron oxide particles at the head and platinum in the tail part of the fish using microscale continuous optical printing ( $\mu \mathrm{COP}$ ). By using hydrogen peroxide as the fuel, the chemical decomposition reaction of the platinum catalyst and hydrogen peroxide provided the propulsion drive for the microfish at the tail, while it was being steered under the applied magnetic field through the head part of the structure.[31] Shao et al. devised a complex micro-scale (less than a millimeter diameter) structure coil spring using iron oxide incorporated PEGDA hydrogel with continuous liquid interface production (CLIP) technique. UV light was used to cure a vat of hydrogel. Although complex magnetically-active structures were achieved, structures were not characterized as actuators.[32] Cilium of PDMS-iron oxide 
nanoparticles, was 3-D printed by ink writing of PDMS-iron oxide nanoparticles and their bending actuation were studied under the permanent magnetic field.[33] Roh et al. 3-D printed smart mesh-like structure of PDMS and iron microparticles that floated on the liquid surface. Under an applied magnetic field, these structures underwent up to $20 \%$ strain. Moreover, their application as a soft robot that can grab and transport particles on the liquid interface under alternating magnetic field was shown.[34] Kim et al. developed complex structures with direct ink writing of silicon-based material while using a secondary fugitive support ink as the wall for confining the magnetic silicon ink. During the extrusion, alternating magnetic was applied to the nozzle tip to align the magnetic $\mathrm{NdFeB}$ microparticles in predefined directions. As the silicone curing completed, the support wall was washed away. Alignment of the magnetic particles in different directions enabled complex fast shape change of the soft structure as well as locomotion and transport of the cargo under the magnetic field.[35]

Although the employment of 3-D printing for the fabrication of soft actuators was recently noticed, it still didn't has not reached its full potential as most of these 3-D printed structures still lacks the complexity and simplicity of the process now common with fused filament fabrication (FFF) the most popular type of 3-D printing. In addition, while some of the studies enabled the fabrication of complex structures as coils, the 3-D printing method that were used are expensive and limit accessibility for both researchers as well as commercialization. On the other hand, while the use of support material enabled fabrication of more complex soft actuators, they still lack the potential to fabricated complex arbitrary structures as a helical coil that lacks support from previously printed layers as in 
conventional printing. Since most of these soft actuators are comprised of silicon-based elastomeric composites, they require curing at a higher temperature for an extended time. As the curing process is time-consuming, these silicon-based structures tend to lose shape easily due to the lack of support.

In order to overcome these challenges, embedded 3-D printing methods were introduced to develop free-form complex structures.[36-38] In these methods, structures are printed inside a secondary hydrogel bath that provides the temporary support for the printed structures. Successful fabrication of electrically conductive soft helical coil structures of PDMS- carbon nanotube was achieved in another work by the author.[39] Here, we developed complex helical coil magnetic soft actuators of PDMS-iron oxide nanoparticles using these embedded 3-D printing methods. Proper design of helical coil parameters, material composition and chose of printing bath achieved successful fabrication of helical magnetic coil structure. Capability of soft helical coil structure in achieving high linear actuation and bending actuation is demonstrated. The effect of iron oxide concentration on the actuation behavior of these 3-D printed samples is studied. The advantages of helical coil structure for locomotion under applied magnetic field for soft robot and their ability to climb a vertical surface is shown and discussed. 


\section{Chapter 2: Materials and Methods}

\subsection{Materials}

Iron oxide $\left(\mathrm{Fe}_{3} \mathrm{O}_{4}\right)$ with a diameter of $15-20 \mathrm{~nm}$ was purchased from the U.S. Research Nanomaterial Lab (Houston, TX). Pluronic f-127 powder was purchased from Sigma-Aldrich (St. Louis, MO). Chloroform was purchased from Fisher Scientific (Waltham, MA). PDMS Slygard 182 was purchased from DOW Corning (Midland, MI).

\subsection{Preparation of PDMS- Iron Oxide Nanoparticle Composite}

Iron oxide nanoparticles (NP) were dispersed in the PDMS using ultrasonication. To enhance the dispersion of the nanoparticles, chloroform was used as the solvent. First, each of the PDMS part A and iron oxide nanoparticles were separately dispersed in chloroform by bath sonication for 1 hour. For each 3 grams of PDMS part A, $20 \mathrm{ml}$ of chloroform was used. To enhance the dispersion of the magnetic particles, for each 0.3 gram of iron oxide $20 \mathrm{ml}$ of chloroform was used (maximum of $1.5 \% \mathrm{~W} / \mathrm{V}$ ratio of iron oxides to chloroform). Then, dispersions of PDMS-chloroform and iron oxide NP-chloroform were mixed together by probe ultrasonication. Probe sonication was done for 1 hour, with 1 second on and 1 second of pulse at $80 \%$ frequency. Finally, the dispersion was then heated on a hotplate set at $120{ }^{\circ} \mathrm{C}$ so that the chloroform evaporated as fast as possible, where the dispersion of iron oxide NP in PDMS was achieved. Then, PDMS part B (curing agent) wax mixed with the prepared dispersion with a ratio of $1: 10$. After carefully mixing by for 15 minutes, the prepared ink sat for $1 \mathrm{~h}$ in room temperature for making sure that trapped gasses were removed. 


\subsection{Preparation of Pluronic Hydrogel}

The Pluronic f-127 hydrogel was made with $40 \%$ weight concentration. The pluronic powder was gradually added to a container of deionized water (DIW) at $4{ }^{\circ} \mathrm{C}$ that placed in the ice bath, where it was being magnetically stirred at $700 \mathrm{rpm}$. Ice bath temperature was controlled by adding ice until all the powder was dissolved. This process might take hours depending on the volume of the hydrogel being prepared (up to 24 hours for $500 \mathrm{ml}$ ). After complete dissolution, the hydrogel was stored in refrigerator for further use.

\subsection{3-D Printing}

The materials were 3-D printed on an open-source converted Lulzbot Mini (Aleph Objects, Loveland CO). The mini was adapted with an open-source syringe pump.[40] The fused filament fabrication (FFF) nozzle extruder was removed and the 3-D printed syringe pump was mounted in its place. In order to have the printer functioning properly, start and end G-code was removed, and a line of G-code was added for each print so that the software enables cold extrusion at room temperature (M301 P2 ).

For the fabrication of complex helical coil structures, an embedded 3-D printing method was used in which the structure is printed inside a secondary hydrogel bath to provide temporary support for the structure. The pluronic hydrogel was used as the secondary bath. To achieve successful printing of miniature helical coil structures with diameter of $4 \mathrm{~mm}$, a custom G-code was written to define the helical print path. To achieve fine resolution, 1-inch long gauge 29 blunt needles with an inner diameter of 180 micrometers was utilized on a $3 \mathrm{ml}$ Luer-lok syringe. Long needle enables reaching out to 
higher bath depth without disturbing the gels and prints. Prior to the start of each print, a few milliliters of $4{ }^{\circ} \mathrm{C}$ pluronic (pluronic in liquid state) was added to the top of the hydrogel container so that the liquid pluronic fills the possible voids that might be created during the print process. As the printing completed, the printing containers were confined with parafilm and put on the hotplate at $85{ }^{\circ} \mathrm{C}$ for curing. Inks with a higher concentration of iron oxide required longer curing time. As the curing completed, the container was cooled down to for the pluronic to turn into liquid and the printed structures were released. Finally, printed coils were removed and were washed with $4{ }^{\circ} \mathrm{C}$ DIW to remove pluronic residuals.

Table 1. 3-D printing parameters.

\begin{tabular}{|c|c|c|c|c|}
\hline $\begin{array}{c}\text { Iron oxide } \\
\text { concentration (\%) }\end{array}$ & $\begin{array}{c}\text { Needle } \\
\text { gauge }\end{array}$ & $\begin{array}{c}\text { Print speed } \\
(\mathbf{m m} / \mathbf{s})\end{array}$ & $\begin{array}{c}\text { Extrusion } \\
\text { rate }\end{array}$ & $\begin{array}{c}\text { Curing time } \\
\text { (h) at } \mathbf{~ s 5}^{\circ} \mathrm{C}\end{array}$ \\
\hline 10 & 30 & 2 & 0.08 & 16 \\
\hline 20 & 30 & 2 & 0.09 & 18 \\
\hline 30 & 30 & 2 & 0.13 & 22 \\
\hline
\end{tabular}

\subsection{Characterization}

\subsubsection{Printing Material Rheometry}

The 3-D printing materials were characterized using a HR-2 rheometer (TA Instruments). Constant volume of the 3-D printing materials $(1 \mathrm{ml})$ was put under the device with a cone of $20 \mathrm{~mm}$ diameter and characterization were completed on the 
amplitude sweep mode with the strain of 0.1-500 for 25 data point, where storage and loss modulus were measured.

\subsubsection{Microscopic imaging}

Field emission scanning electron microscopy (FESEM) (SE-4700 Hitachi) was utilized to investigate the morphology of the prepared composites. Images with magnifications of $5-40 \mathrm{KX}$ captured at $5 \mathrm{KV}$ voltage were used to determine the efficacy of particles dispersion method and formation of agglomeration.

\subsubsection{Atomic force microscopy}

AFM (Dimension ICON system) (Bruker, CA.) was employed to study surface characteristics of the $3 \mathrm{D}$ printed composites under ambient conditions. AFM provides the surface topology of the printed composite. Peaks and roughhouses on the surface can be attributed to embedded nanoparticles which change surface characteristics.

\subsubsection{Linear and bending magnetic actuation test}

To study the bending actuation performance, helical coil structure with known length were hung from one end, while three constant N42 magnet (K\&J Magnetics, Pipersville, PA) with $19 \mathrm{~mm}$ diameter and $6.5 \mathrm{~mm}$ thickness with surface field of 3661 Gauss were stacked together, that provided a surface field of 5902 Gauss for the three stacked magnets. The stacked magnets were set on a magnet holder stage and stationed at $40 \mathrm{~mm}$ distance with theirs poles perpendicular to the free end of the hanging structure. The magnets were then moved toward the structure on known intervals with the use of a manipulator and the imaging completed for each step. Similarly, to characterize linear 
actuation, the magnets were positioned at a known distance with their pole aligned with the vertical axis of the hanged coil structure. Imaging completed at a known interval of $5 \mathrm{~mm}$ by moving the magnets toward the structure, using ImageJ software, deformation was measured.

\subsubsection{Coil Locomotion Tests}

A thin (1/16 inch) clear acrylic plate was used as the substrate. Cameras were positioned to capture the top and side views. By moving a magnet under the structure, coil structures began to rotate where the locomotion was captured. Making incline surfaces at known intervals of 10 degrees, coil structure effectiveness to perform as the soft robots on the upward direction were investigated. 


\section{Chapter 3: Results}

\subsection{3-D Printing Results}
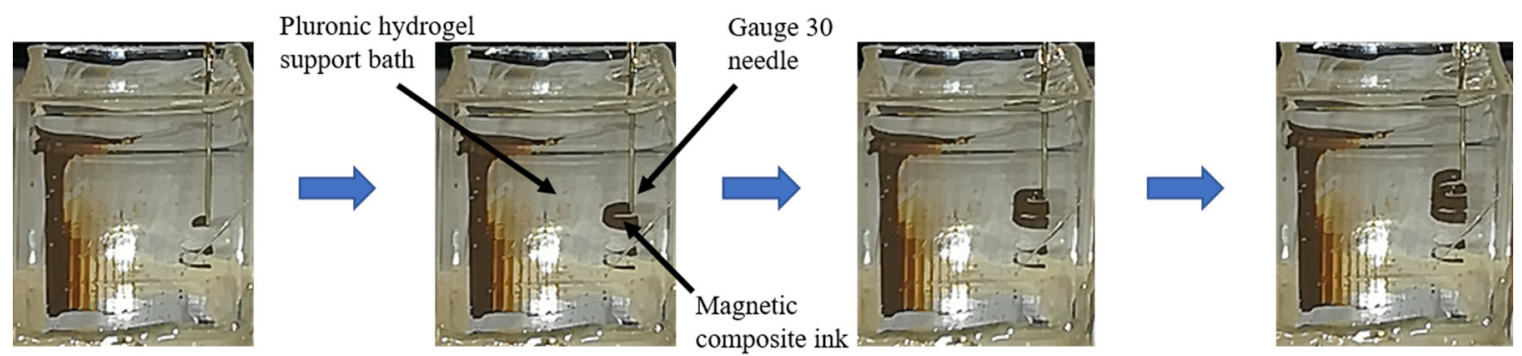

Figure 1.- Photographs illustrating the 3-D printing process of helical coils of PDMS-magnetic iron oxide nanoparticles.

3-D printing of the helical coil structures (coil diameter of $4 \mathrm{~mm}$, pitch of $1.6 \mathrm{~mm}$ ) was done through the embedded printing method as described in the material and methods section, where pluronic f-127 was used as the secondary support bath to provide the temporary support duning fabrication of geometrically complex models. Figure 1 shows the printing process of a helical coil structure. Printing starts as the long needle reaches the depth of the hydrogel bath. As material is injected, the shear stress originated by needle's movement tums the hydrogel to liquid surrounding the needle's tip, where the composite is injected. As the needle translates away from a specific voxel in the bath, the shear stress is removed from that volume that will result in the liquefied hydrogel retuming to the oniginalgel state and confining the extruded magnetic material. Thus, the high viscosity pluronic hydrogel provides the required support for the next voxel (e.g as shown in Figure 1 the tums of the coil to be injected) in the design path and prevents it collapsing to the next layer and nuining the print (Figure 2). As the printing is completed, the printed structure is set on the hotplate at $85^{\circ} \mathrm{C}$ until the curing of the structure. It should be noted 
that although pluronic is a hydrogel with $60 \%$ water content, it holds its form in relatively high temperatures needed for curing for hours.
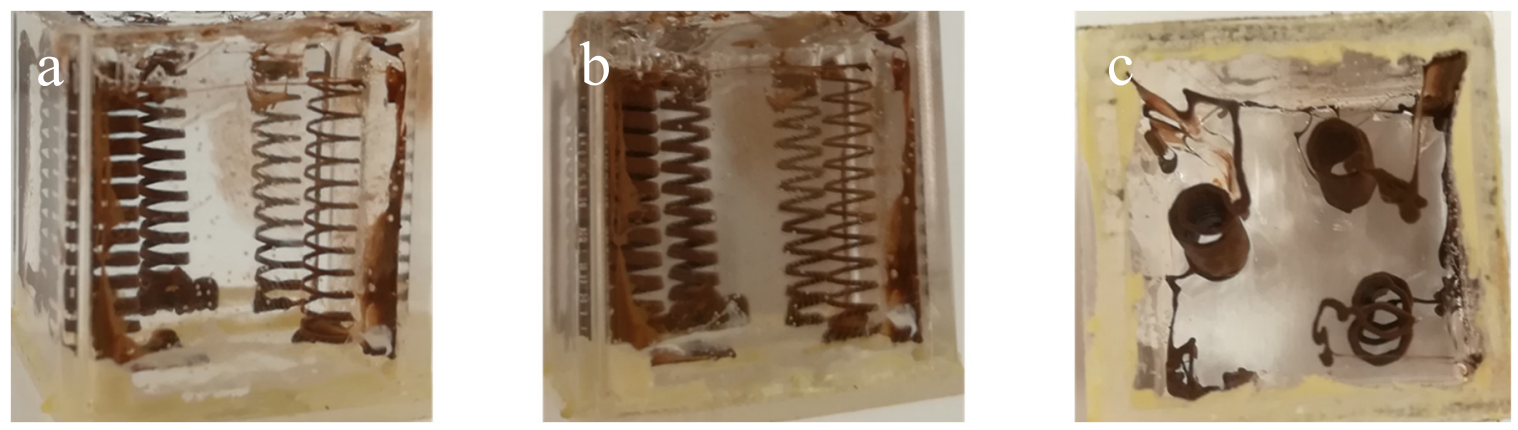

Figure 2. Completed 3-D printed magnetic helical coil structures inside pluronic hydrogel support bath. a \& b) Side view of 3-D printed coils printed with various injection rate. c) Bottom view of the cross section of 3-D printed helical coil inside the pluronic support bath.
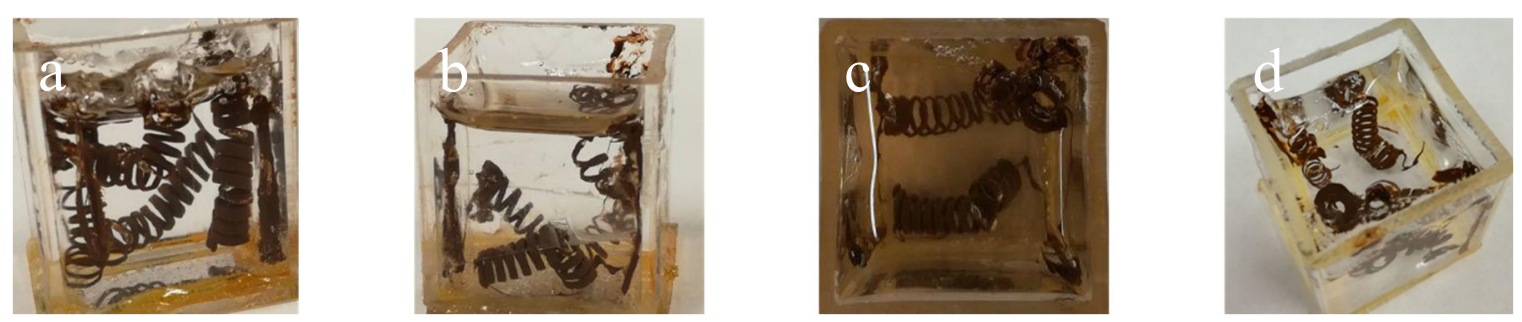

Figure 3- Release of cured 3-D printed PDMS-iron oxide composites after reducing the hydrogel bath temperature to $4^{\circ} \mathrm{C}$, a \&b) side view of released 3-D printed helical coils, c \& d) Top view of 3-D printed helical coil released after completion of curing.

3-D prints are released from the bath by returning the hydrogel to $4{ }^{\circ} \mathrm{C}$, where the pluronic hydrogel turns into liquid and release the printed structure (Figure 3).

\subsection{Ink rheometry:}

Loss modulus can be defined as the dissipated amount of energy during the oscillatory shear flow, G”. Storage modulus, G', can be defined as the amount of energy stored in the viscoelastic liquid during the oscillatory shear flow.[41] Loss modulus is an indication of 
the viscous behavior of the material and storage modulus is an indication of elastic behavior of the material. These material properties are measured by oscillation rheometry technique for the prepared composites with three different concentration ratios and the results are shown in Figure 4. As can be seen in Figure 4, for each concentration ratio, the loss modulus is higher than the storage modulus. The loss modulus for the composite for the $30 \%$ filler nanoparticle composite is almost one order of magnitude higher than loss modulus for the $10 \%$ iron oxide filler ratio composite. However, all the formulated composite inks have a viscosity (loss modulus) close to the pure PDMS.

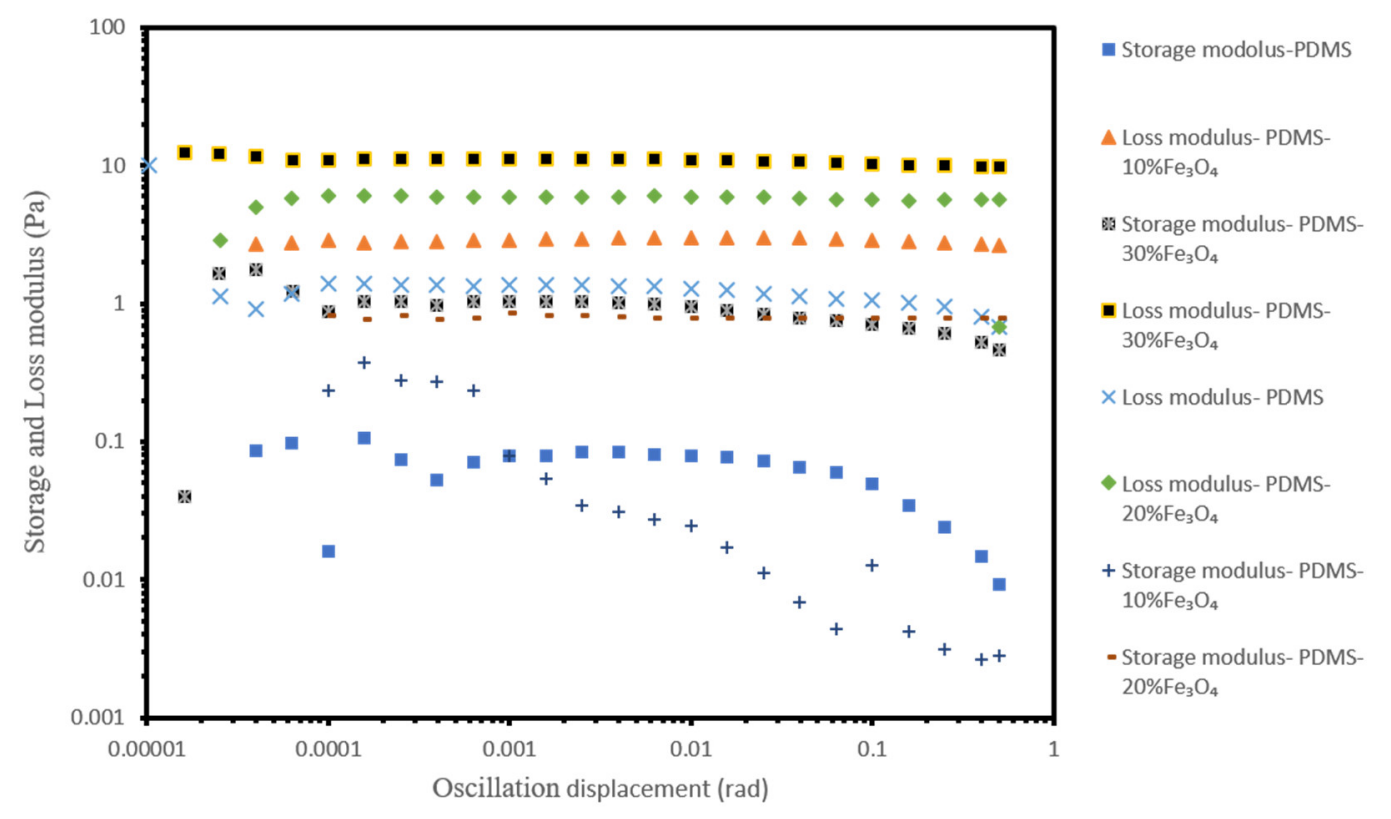

Figure 4- Ink rheometry: Measure loss and storage modulus (G' and G respectively ) for three different concentration of PDMS- iron oxide nanoparticle filler. 


\subsection{SEM and AFM images}

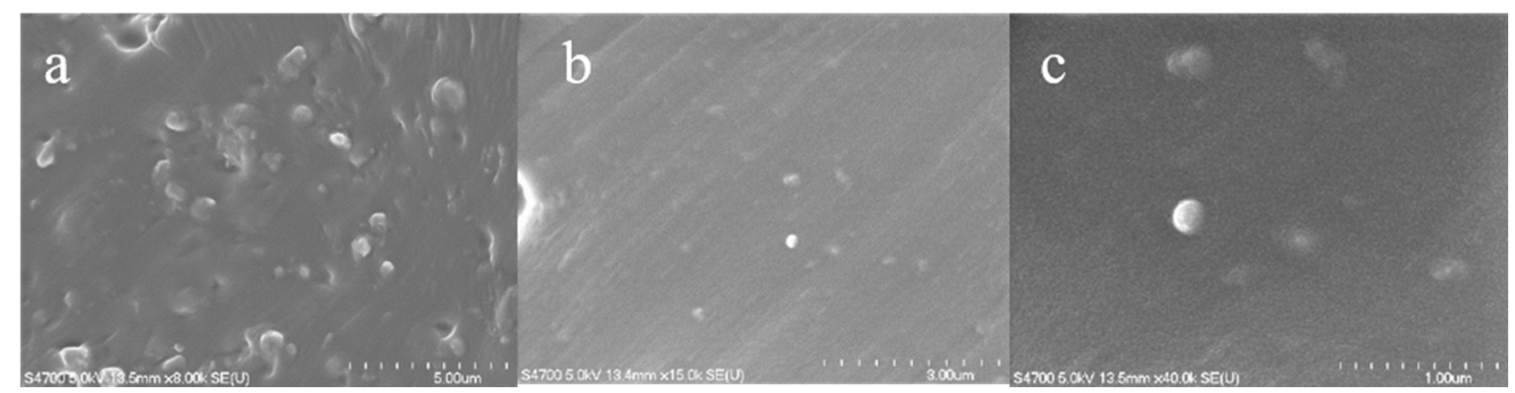

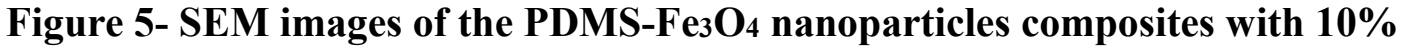
concentration at $8 \mathrm{~K}(\mathrm{~A}), 15 \mathrm{~K}(\mathrm{~B})$, and $(40 \mathrm{~K})$ magnification, depicting agglomeration of particle in size of $50-500 \mathrm{~nm}$.
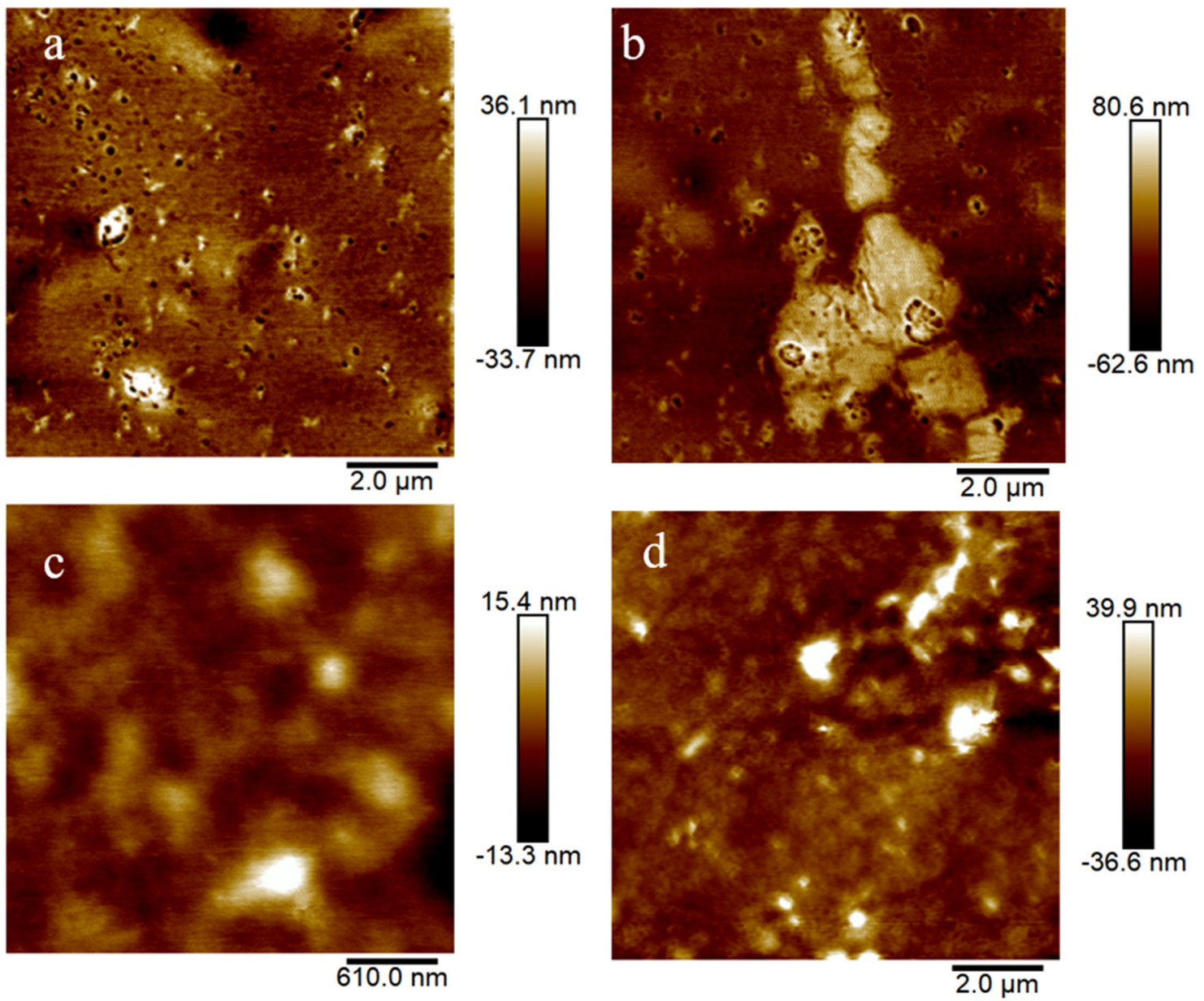

$39.9 \mathrm{~nm}$

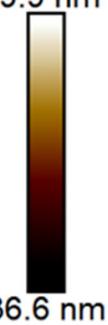

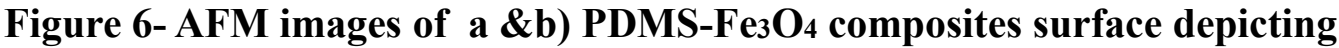
nanoparticles aggregates, c \& d) AFM images of pure PDMS 
Cross-sectional FESEM images depict a relatively scarce dispersion of nanoparticle filler inside the PDMS polymer matrix as seen in Figure 5. Particles size are varied between $50 \mathrm{~nm}$ to $500 \mathrm{~nm}$. However, the smaller single spherical nanoparticles of iron oxide with a nominal diameter of 15-20 nm was not detected. While there are that studies claimed very fine dispersion of nanoparticles using similar dispersion methods with iron-oxide microparticle where SEM images showed a very homogenous dispersion. That could be possible that the fact that microparticle was used instead help in better monodispersion of particles compared to nanoparticles.[23, 33, 35] AFM provides high resolution images of the composite's surface and provides a better determination of surface morphology than FESEM. Figure 6 shows a typical AFM of the composite's surface, where smaller single spherical particles can be observed throughout the surface along with larger particles. Larger particles span up to half a micrometer, implying large aggregate of nanoparticles. While bright dot light can be observed in both type of composites, the surface roughness in pure PDMS relatively smaller (less than 20nm), the surface roughness in composites are as high as $80 \mathrm{~nm}$ which could be aggregate of the nanoparticles as the SEM images suggest. 


\subsection{Magnetic Actuation Testing}

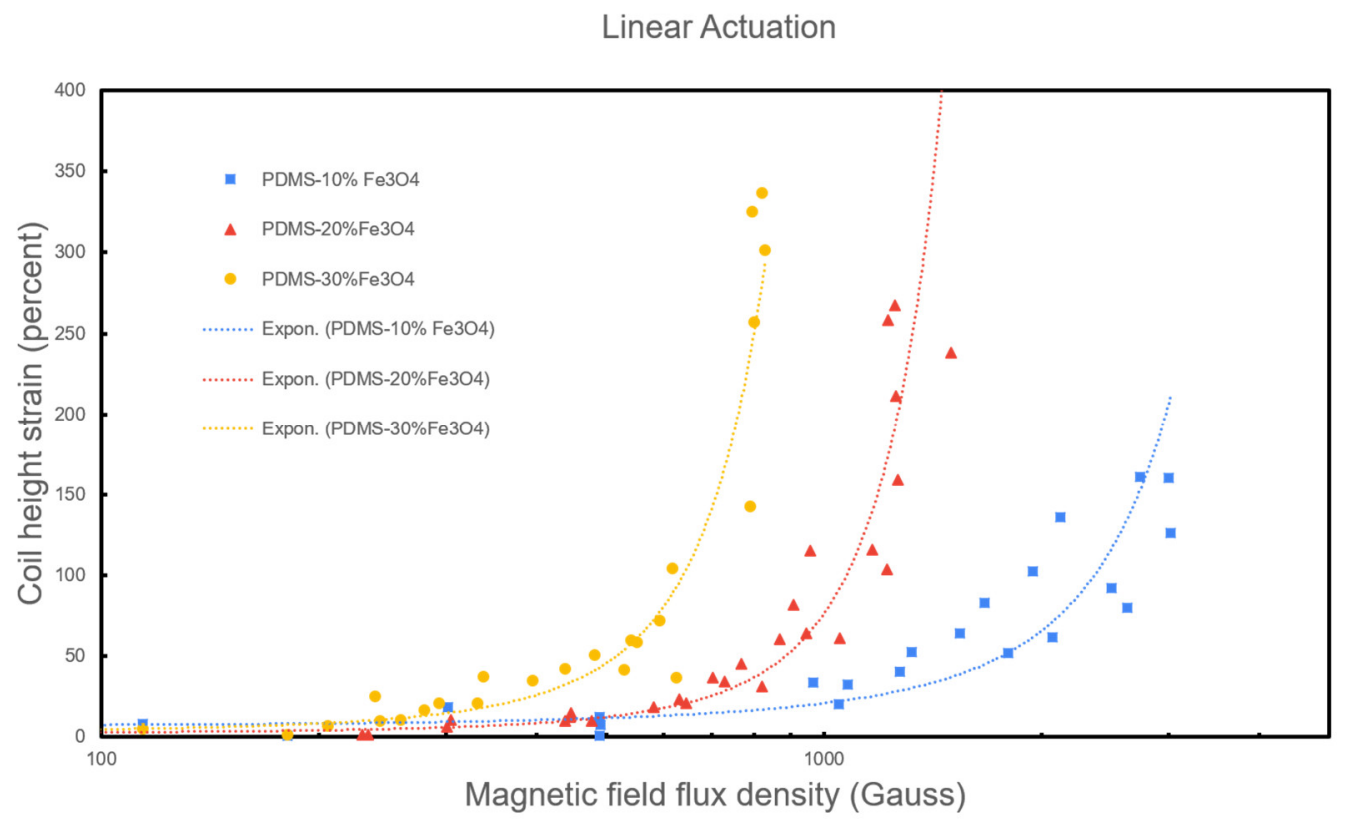

Figure 7- Linear actuation behavior of the helical coil structures with 10, 20, and, $30 \% \mathrm{Fe}_{3} \mathrm{O}_{4}$ nanoparticles embedded in PDMS matrix under applied magnetic field.

3-D printed helical coil structures characterized as linear magnetic actuators. Linear actuation was defined as a change in the initial structure's height under an applied magnetic field divided by the initial structure's height. While increasing the magnetic field strength, the coils behaved in a semi-linear pattern until they reach a certain limit where forces exert the coils springs' restoring forces. Then the coils completely unwind and get attached to the magnet. This actuation consist of both straightening the coil's fiber and fiber's stretching. Increasing the magnetic particle weight ratio greatly increased actuation performance. While for $10 \%$ iron oxide weight ratio a field of 3000 Gauss was needed to unwind the coils to achieve a $160 \%$ actuation, $20 \%$ and $30 \%$ weight ratio composite maximum actuation of $267 \%$ and $337 \%$ was achieved with a magnetic field of 1125 and 820 Gauss, respectively (Figure 7). Thirty percent iron oxide embedded composites 
attained the largest deformation under an applied magnetic field, specifically under smaller magnetic field strength where actuators perform in a linear manner.

\section{Bending Actuation}

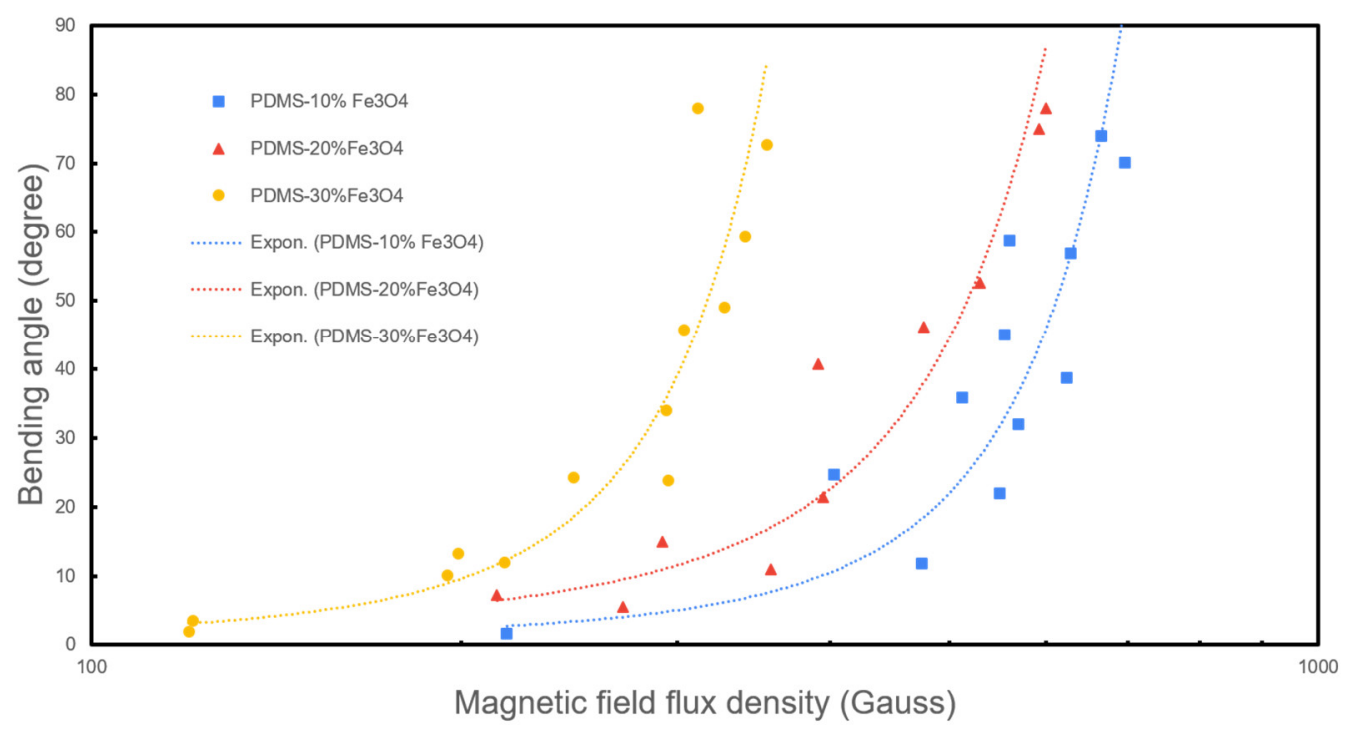

Figure 8- Bending actuation behavior of the helical coil structures with 10, 20, and, $30 \% \mathrm{Fe}_{3} \mathrm{O}_{4}$ nanoparticles embedded in PDMS matrix under applied magnetic field.

Bending actuation behavior of 3-D printed helical coil under an applied magnetic field was studied for three different iron oxide concentration composites. The bending actuation also showed a semi-linear and exponential behavior like linear actuation. The coils showed a linear increase in the bending up to $25^{\circ}, 22^{\circ}$ and $21.8^{\circ}$ for $30 \%, 20 \%$ and 10\% iron oxide embedded PDMS helical coils under field of 250, 400 and 550 Gauss. After that, coils started straightening and reaching and as the result bending angle increased exponentially, reaching maximum of $80^{\circ}, 78^{\circ}$ and $72^{\circ}$ for $30 \%, 20 \%$ and $10 \%$ iron oxide embedded PDMS coils at field with intensity of 312, 600, 700 Gauss respectively. 


\section{Chapter 4: Discussion}

To achieve successful helical coil structures of the PDMS-iron oxide nanoparticles composite, embedded 3-D printing was utilized to enable fabrication of a structure that lacks the typical support provided by the just printed beneath layer in typical fused filament fabrication (FFF) and fused deposition modeling (FDM) prints. The helical coil structure's printing was done in a single continuous extrusion of the composite material into the support bath. The design of the hydrogel support bath was critical to achieve a successful print; pluronic turns into a liquid at below $10{ }^{\circ} \mathrm{C}$ temperature and stays at gel state at temperatures above. As a result, the hydrogel provided the required support for the structure that prevents it from collapsing due to gravity for extended hours at high temperatures that is essential for facilitating PDMS curing. To avoid the introduction of air voids inside the bath as the result of needle movement, a small quantity of liquid pluronic (pluronic at a temperature below $4{ }^{\circ} \mathrm{C}$ ) was provided at top of the bath to fill the gaps while completing the print process. This was especially helpful during the curing process as the extended heating cause the trapped air to expand and could potentially ruin the prints. As the curing of the PDMS was completed, the pluronic bath temperature was lowered to $4{ }^{\circ} \mathrm{C}$ to liquefy. When pluronic liquefied, printed coil structures were released from the bath. Moreover, pluronic provided higher resolution and smaller feature sizes compared to the other types of support bath such as gelatin for the PDMS material. [39]

Although other studies attempted fabrication of helical coil structures using a similar mechanism, they failed to achieve fabricating free-standing coils.[37, 38, 42] While some 
of these works were able to release the cured structure from the hydrogel support bath, however, they only kept their shapes inside a liquid phase and no reports of successful freestanding coils in the air were given. An imperative factor in achieving successful prints was the right coil design parameters. A variety of print speeds were attempted while keeping other parameters (structure geometry, extrusion rate, needle size) the same. A faster speed provided a smaller fiber's diameter, while reducing print speed increases that. As the result, with gauge 29 needles, $2 \mathrm{~mm} / \mathrm{s}$ print speed was selected. This print speed allowed enough time for the material to be extruded throughout the print pass homogenously, yielding fiber's with diameter of 0.8-1.2 $\mathrm{mm}$, while final coil's diameter varied between 3.6-4.3 $\mathrm{mm}$. As the ratio of the fiber's diameter to the coil's diameter decreased, 3-D printed coil structures lost their shape after removal from the bath by unwinding and turning into a straight fiber while immersing them into a liquid like DIW retained them their coil geometry.

Rheometry tests of ink gave insight into the reasonable formulation of the composites to achieve desirable printability. Increased viscosity requires a larger needle gauge to print ,which dramatically affects the resolution of the prints. $10 \%$ and $20 \%$ iron oxide concentrations yielded inks with similar loss modulus to the plain PDMS. Loss modulus G" can be interpreted as the viscous behavior of the material.[41] Since both concentrations had similar loss modulus to the PDMS, printing with a small gauge 30 needle (180 micrometers inner diameter) was achieved. In the case of the PDMS with $30 \%$ iron oxide nanoparticles, although the loss modulus was almost twenty times higher than pure PDMS, the material still had more of a liquid like behavior than the gel behavior and it was possible 
to print with the same gauge 30 needles[43]. However, a slightly higher extrusion rate was required to compensate for the increased viscosity. These results indicate that future work could achieve even higher resolutions with these materials.

One of the key advantages of embedding iron oxide particles with $15-20 \mathrm{~nm}$ size was that they only slightly increased viscosity even up to $30 \%$ weight embedded nanoparticles. Hence, formulated 3-D printing materials were still printable with fine 180 -micrometer diameter needles that enable high resolution, whereas in other studies extrusion printing silicon-based magnetic particle incorporated ink needles with a diameter of $410 \mathrm{um}$ had been used.[35]

Achieving successful and homogeneous dispersion of nanoparticles inside the matrix is critical for attaining a composite's 3-D printable multifunctionality. Morphology of the synthesized composite was studied using FESEM and AFM. FESEM depicted a PDMS matrix embedded with few aggregated particles of size between $50 \mathrm{~nm}$ to $500 \mathrm{~nm}$, while AFM provided a better insight into the nanoparticle's dispersion with higher resolution. Comparing the AFM images of the composite's surface to the pure control PDMS sample, similar small bright spot-like throughout the sample was observed suggesting that those bright spots cannot be considered the fine dispersion of iron oxide nanoparticles with diameter of 15-20 nm. However, formation of aggregate of iron oxide particles can be noticed. Considering both AFM and FESEM images, it can be suggested that the dispersion mechanism was not effective in achieving monodispersed nanoparticles. (Figure 5 \& 6)

3-D printed helical coil structures were characterized as linear magnetic actuators with $30 \%$ embedded iron oxide composites achieving the highest actuation. Coil actuator 
devices demonstrated a linear actuation behavior under the applied magnetic field until they reach a certain threshold where the magnetic pull force overcomes the restoring force. In the linear region, the maximum achieved linear actuation was $50 \%, 60 \%$ and $75 \%$ for coils of 10, 20, and 30\% magnetic particle embedded devices, respectively. As devices passed the linear region, they suddenly undergo large deformation where coils completely unwind and enable a large stroke, from $150 \%$ of initial to $360 \%$. This noticeable actuation is achieved due to the unique design of the helical coil structure device through 3-D printing fabrication method. No other reports of soft 3-D printed magnetic linear actuation are available to the best of our knowledge. Several studies fabricated dielectric elastomer actuator using microfabrication techniques where strains from $2 \%$ up to $150 \%$ were achieved. These dielectric elastomer actuators required a very high voltage (electric field) to operate.[44-46] Yu et al. reported a bistable electroactive polymer actuator where a maximum actuation of $80 \%$ at $2400 \mathrm{~V}$ and $70{ }^{\circ} \mathrm{C}$ condition was achieved.[47] Tawk et. al 3 -D printed a negative pressure (vacuumed) soft actuator where a maximum of $250 \%$ compression was achieved, while they required order of magnitudes more time (60-90s) compared to the magnetic actuator to operate[48].

Magnetic bending actuation characterization of the 3-D printed coils demonstrated noticeable bending properties of the helical structures achieving up to $80^{\circ}$ bending angle. While $30 \%$ embedded iron-oxide nanoparticle embedded composites achieving $80^{\circ}$ bending actuation with an applied field of 312 Gauss, $20 \%$ and $10 \%$ embedded composites achieved bending of $78^{\circ}$ and $72^{\circ}$ at field of 600 and 700 Gauss respectively. Similar to linear actuation, bending behavior also demonstrated a semi-linear actuation performance 
region at the beginning while increasing exponentially with stronger fields that makes the coil's fiber straighten.

Application of 3-D printed helical coil structures for untethered soft robots under applied magnetic field was demonstrated for the $10 \%$ embedded iron-oxide nanoparticles composites. Locomotion of soft robots on inclines are challenging as the need to withstand their own weight as they displace. The larger the incline angle the robots can successfully travel on, exhibits the better suitability of them for untethered soft robot application. $10 \%$ embedded iron oxide nanoparticles successfully travel on inclines of $45^{\circ}$. Moreover, it was shown that they can easily climb vertical surface. Park et al. fabricated helical coils of PDMS embedded iron-oxide particles through molding and characterized it as untethered soft robots, where they demonstrated limited displacement of $20 \mathrm{~cm}$ on a small incline of $15^{\circ} \cdot[49]$

PDMS is a widely used biocompatible material in biomedical and microfluidic industries that have been used for various applications.[50] As a magnetic field is applied remotely and magnetic actuators possess fast response, the 3-D printed helical coil actuator can be used as the artificial muscle.[51] Moreover, these coils can be employed for catheter guidance or tissue manipulation applications.[52, 53] Also, miniature helical coils have been utilized as the leg for robots.[49] Enhancing the dispersion of the magnetic nanoparticles along the length of the 3-D print can potentially enhance the performance of the actuators. Increasing the particle size improves the magnetic performance, however, negatively effects the viscosity of the printing material and printing resolution. Optimizing particle size in regard of the printing material viscosity has the potential to enhance 
actuation performance and is left for future work. In addition, by using a two head printer the density of magnetic nanoparticles could be varied created more complicated actuation. Finally, incorporation of conductive particles along with magnetic particles could improve multifunctional composites that can be used for applications such as antenna or magneto resistive sensors in 3-D printed soft electronics.[54, 55] 


\section{Conclusion}

This study demonstrated how to successfully 3-D print complex soft magnetic actuators of PDMS-iron oxide nanoparticles with an embedded printing method. Employment of pluronic $\mathrm{f}-127$ hydrogel as the support bath and maintaining the proper ratio between fiber's diameter to coil's diameter enabled successful fabrication and removal of free-standing helical coil springs. Utilizing iron oxide nanoparticles with 15-20 $\mathrm{nm}$ diameter preserved viscosity of the composite's close to pure PDMS, which facilitated achieving high resolution through the employment of gauge 29 (180 micron diameter) needles. The development of complex helical coil structures enabled linear magnetic actuation, which demonstrated a noticeable $360 \%$ device's linear actuation and $80^{\circ}$ bending actuator in helical coils containing 30\% iron oxide nanoparticles. Printed helical coil structure application as untethered soft robot was demonstrated where they were able to easily be steered on $45^{\circ}$ and $90^{\circ}$ inclines. PDMS- based helical coils can be employed as in a variety of applications such as biocompatible artificial muscles and to catheter steering systems or fast rotating legs for soft robots. 


\section{Reference List}

1. Janocha, H., Actuators. 2004: Springer.

2. Mirvakili, S.M. and I.W. Hunter, Artificial muscles: Mechanisms, applications, and challenges. Advanced Materials, 2018. 30(6): p. 1704407.

3. Lee, C., et al., Soft robot review. International Journal of Control, Automation and Systems, 2017. 15(1): p. 3-15.

4. Kongahage, D. and J. Foroughi, Actuator Materials: Review on Recent Advances and Future Outlook for Smart Textiles. Fibers, 2019. 7(3): p. 21.

5. Cao, X., et al., Review of soft linear actuator and the design of a dielectric elastomer linear actuator. Acta Mechanica Solida Sinica, 2019. 32(5): p. 566579.

6. Zolfagharian, A., et al., Evolution of $3 D$ printed soft actuators. Sensors and Actuators A: Physical, 2016. 250: p. 258-272.

7. $\quad$ Sells, E., et al., RepRap: the replicating rapid prototyper: maximizing customizability by breeding the means of production, in Handbook of Research in Mass Customization and Personalization: (In 2 Volumes). 2010, World Scientific. p. $568-580$.

8. Jones, R., et al., RepRap-the replicating rapid prototyper. Robotica, 2011. 29(1): p. 177-191.

9. $\quad$ Bowyer, A., 3D printing and humanity's first imperfect replicator. 3D printing and additive manufacturing, 2014. 1(1): p. 4-5.

10. Pearce, J.M., N. Anzalone, and C. Heldt, Open-source wax RepRap 3-D printer for rapid prototyping paper-based microfluidics. Journal of laboratory automation, 2016. 21(4): p. 510-516.

11. Anzalone, G.C., B. Wijnen, and J.M. Pearce, Multi-material additive and subtractive prosumer digital fabrication with a free and open-source convertible delta RepRap 3-D printer. Rapid Prototyping Journal, 2015. 21(5): p. 506-519.

12. Holland, D.P., et al., The soft robotics toolkit: Strategies for overcoming obstacles to the wide dissemination of soft-robotic hardware. IEEE Robotics \& Automation Magazine, 2017. 24(1): p. 57-64. 
13. Morrow, J., S. Hemleben, and Y. Menguc, Directly fabricating soft robotic actuators with an open-source 3-D printer. IEEE Robotics and Automation Letters, 2016. 2(1): p. 277-281.

14. Saito, A., et al. RepRap SWIM-ER: low cost open-source 3D gel printer. in Nano, Bio-, Info-Tech Sensors, and 3D Systems II. 2018. International Society for Optics and Photonics.

15. Sato, K., et al. Development of low-cost open source 3D gel printer" RepRap SWIM-ER". in Nanosensors, Biosensors, Info-Tech Sensors and 3D Systems 2017. 2017. International Society for Optics and Photonics.

16. Gul, J.Z., et al., 3D printing for soft robotics-a review. Science and technology of advanced materials, 2018. 19(1): p. 243-262.

17. Taylor, A.P., et al., Fully 3D-Printed, Monolithic, Mini Magnetic Actuators for Low-Cost, Compact Systems. Journal of Microelectromechanical Systems, 2019. 28(3): p. 481-493.

18. Janocha, H., Application potential of magnetic field driven new actuators. Sensors and Actuators A: Physical, 2001. 91(1-2): p. 126-132.

19. Xu, X., et al., Self-sensing, ultralight, and conductive $3 D$ graphene/iron oxide aerogel elastomer deformable in a magnetic field. ACS nano, 2015. 9(4): p. 39693977.

20. Song, $\mathrm{K}$. and Y. Cha, Fe3O4-silicone mixture as flexible actuator. Materials, 2018. 11(5): p. 753.

21. Darmawan, B.A., et al. Simple fabrication of helical-shaped microrobot with NdFeB nanoparticle by self-rolling technique. in 2019 International Conference on Manipulation, Automation and Robotics at Small Scales (MARSS). 2019. IEEE.

22. Venkiteswaran, V.K., et al., Bio-Inspired Terrestrial Motion of Magnetic Soft Millirobots. IEEE Robotics and automation letters, 2019. 4(2): p. 1753-1759.

23. Pirmoradi, F.N., L. Cheng, and M. Chiao, A magnetic poly (dimethylesiloxane) composite membrane incorporated with uniformly dispersed, coated iron oxide nanoparticles. Journal of Micromechanics and Microengineering, 2009. 20(1): $\mathrm{p}$. 015032 .

24. Evans, B.A., et al., A highly tunable silicone-based magnetic elastomer with nanoscale homogeneity. Journal of magnetism and magnetic materials, 2012. 324(4): p. 501-507. 
25. Mietta, J.L., et al., Superparamagnetic anisotropic elastomer connectors exhibiting reversible magneto-piezoresistivity. Sensors and Actuators A: Physical, 2013. 192: p. 34-41.

26. Singh, A., et al., Rapid prototyping of magnetic valve based on nanocomposite Co/PDMS membrane. Microsystem technologies, 2014. 20(3): p. 427-436.

27. Lu, H., et al., A bioinspired multilegged soft millirobot that functions in both dry and wet conditions. Nature communications, 2018. 9(1): p. 3944.

28. Ijaz, S., et al., Magnetically actuated miniature walking soft robot based on chained magnetic microparticles-embedded elastomer. Sensors and Actuators A: Physical, 2019: p. 111707.

29. Song, H., et al., Inkjet printing of magnetic materials with aligned anisotropy. Journal of Applied Physics, 2014. 115(17): p. 17E308.

30. Zhang, F., et al., Magnetic programming of 4D printed shape memory composite structures. 2019, Elsevier.

31. Zhu, W., et al., 3D-printed artificial microfish. Advanced materials, 2015. 27(30): p. 4411-4417.

32. Shao, G., et al. High-resolution 3D printing magnetically-active microstructures using micro-CLIP process. in Nano-, Bio-, Info-Tech Sensors and 3D Systems III. 2019. International Society for Optics and Photonics.

33. Liu, F., et al., Fabrication and characterization of a magnetic micro-actuator based on deformable Fe-doped PDMS artificial cilium using 3D printing. Smart Materials and Structures, 2015. 24(3): p. 035015.

34. Roh, S., et al., 3D-Printed Silicone Soft Architectures with Programmed Magneto-Capillary Reconfiguration. Advanced Materials Technologies, 2019. 4(4): p. 1800528.

35. Kim, Y., et al., Printing ferromagnetic domains for untethered fast-transforming soft materials. Nature, 2018. 558(7709): p. 274.

36. Muth, J.T., et al., Embedded 3D printing of strain sensors within highly stretchable elastomers. Advanced Materials, 2014. 26(36): p. 6307-6312.

37. Hinton, T.J., et al., Three-dimensional printing of complex biological structures by freeform reversible embedding of suspended hydrogels. Science advances, 2015. 1(9): p. e1500758. 
38. Zhu, K., et al., Gold nanocomposite bioink for printing 3D cardiac constructs. Advanced functional materials, 2017. 27(12): p. 1605352.

39. Bayaniahangar, R., M.J. Hill, and P.P.S.S. Abadi, 3D Printing of PDMS-CNT Helical Coil Strain Sensor. To be submitted.

40. Wijnen, B., et al., Open-source syringe pump library. PloS one, 2014. 9(9): p. e107216.

41. Han, C.D. and M.S. Jhon, Correlations of the first normal stress difference with shear stress and of the storage modulus with loss modulus for homopolymers. Journal of Applied Polymer Science, 1986. 32(3): p. 3809-3840.

42. Hinton, T.J., et al., 3D printing PDMS elastomer in a hydrophilic support bath via freeform reversible embedding. ACS biomaterials science \& engineering, 2016. 2(10): p. 1781-1786.

43. Almdal, K., et al., Towards a phenomenological definition of the term 'gel'. Polymer gels and networks, 1993. 1(1): p. 5-17.

44. Stoyanov, H., et al., Long lifetime, fault-tolerant freestanding actuators based on a silicone dielectric elastomer and self-clearing carbon nanotube compliant electrodes. Rsc Advances, 2013. 3(7): p. 2272-2278.

45. Corbaci, M., W. Walter, and K. Lamkin-Kennard. Implementation of SoftLithography Techniques for Fabrication of Bio-Inspired Multi-Layer Dielectric Elastomer Actuators with Interdigitated Mechanically Compliant Electrodes. in Actuators. 2018. Multidisciplinary Digital Publishing Institute.

46. Stoyanov, H., et al., Soft conductive elastomer materials for stretchable electronics and voltage controlled artificial muscles. Advanced Materials, 2013. 25(4): p. 578-583.

47. $\mathrm{Yu}, \mathrm{Z}$., et al. Bistable electroactive polymers (BSEP): large-strain actuation of rigid polymers. in Electroactive Polymer Actuators and Devices (EAPAD) 2010. 2010. International Society for Optics and Photonics.

48. Tawk, C., et al., 3D Printable Linear Soft Vacuum Actuators: Their Modeling, Performance Quantification and Application in Soft Robotic Systems.

IEEE/ASME Transactions on Mechatronics, 2019. 24(5): p. 2118-2129.

49. Park, J.E., et al., Magnetomotility of untethered helical soft robots. RSC advances, 2019. 9(20): p. 11272-11280.

50. Peterson, S.L., et al., Poly (dimethylsiloxane) thin films as biocompatible coatings for microfluidic devices: cell culture and flow studies with glial cells. Journal of 
Biomedical Materials Research Part A: An Official Journal of The Society for Biomaterials, The Japanese Society for Biomaterials, and The Australian Society for Biomaterials and the Korean Society for Biomaterials, 2005. 72(1): p. 10-18.

51. Nguyen, V.Q., A.S. Ahmed, and R.V. Ramanujan, Morphing soft magnetic composites. Advanced Materials, 2012. 24(30): p. 4041-4054.

52. Maschke, M., Catheter for magnetic navigation. 2011, Google Patents.

53. Shachar, Y. and L. Farkas, Magnetic linear actuator for deployable catheter tools. 2012, Google Patents.

54. Baharin, R.H.M., et al., Effects of the permittivity and conductivity of human body for normal-mode helical antenna performance. IEICE Electronics Express, 2019: p. 16.20190395 .

55. Satoh, T., P.R. Stauffer, and J.R. Fike, Thermal distribution studies of helical coil microwave antennas for interstitial hyperthermia. International Journal of Radiation Oncology* Biology* Physics, 1988. 15(5): p. 1209-1218. 